\title{
State of the art Nano Materials
}

The special issue of ANM2017 is a collection of papers from the $9^{\text {th }}$ series ANM (Advanced Nano Materials) conference, which was held at the University of Aveiro, Portugal, from 19-21 July 2017.

State of the art results were discussed by eminent researchers on a wide range of subjects including nanomaterials, graphene materials, polymer nanocomposites, magnetic materials, spintronics materials, hydrogen energy, nanoelectronics and solar energy materials. We thank all the reviewers for their detailed reviews which immensely helped the authors to improve the quality of their papers.

Advanced Nano Materials, ANM (https://www.advancednanomaterials-conference.com/) is an annual conference at the University of Aveiro, Portugal and we invite all our colleagues working in the field of energy materials to attend the next ANM.
Guest Editors

Dr.

Elby Titus

TEMA, Department of Mechanical Engineering, University of Aveiro,

Portugal

Prof.

João Campos Gil

Department of Physics, University of Coimbra Portugal

Dr.

Carmen M. Rangel

Laboratório Nacional de Energia e Geologia (LNEG), Portugal E-mail addresses: elby@ua.pt (E. Titus),jmgil@ci.uc.pt (J.C. Gil), carmen.rangel@lneg.pt (C.M. Rangel) 